\title{
\begin{tabular}{|c|lc|}
\hline IV Simpósio Paranaense de Modelagem, & Artigo: & 01 \\
$\begin{array}{c}\text { Simulaçãoe Controle de Processos } \\
\text { ISSN : 1984-7521 }\end{array}$ & Páginas: & $\mathbf{0 1}$ - 08 \\
\hline
\end{tabular}
}

\section{Investigação da Metodologia Clássica de Projeto de Controle para Melhorar o Desempenho Dinâmico do Sistema Fotovoltaico Conectado na Rede}

\author{
Marenice M. de Carvalho, Renan Landau P. de Medeiros e Florindo A. de C. Ayres Junior \\ Universidade Federal do Amazonas, Manaus-AM, Brasil
}

\begin{abstract}
The use of solar panels has been widely adopted as a sustainable alternative for conventional models of power generation. The constant output of photovoltaic systems is not suitable for powering the various devices that use alternative energy as a source. Just as it is not possible to connect to the standard power grid, then the use of DC/AC converters becomes necessary. Also known as a voltage inverter, these devices have the function of converting a constant signal into its input at an alternating signal at the output. In order to connect this generated signal to the electric network it is necessary to guarantee the synchronism of the signals and for this was used the Phase-Locked Loop, PLL, implemented by a PI structure using as a method the place of the roots to determine the gains. A current mode controller was also used to control the power flow from solar generation to the power grid, as the name suggests the controller is activated by a current signal and is also implemented by a PI structure with the cancellation of poles and zeros. Finally, some tests are performed in the developed system to show that with the use of these controllers it is possible to regulate the power flow supplied to the mains while maintaining the phase and frequency of the network.
\end{abstract}

Keywords: Renewable energy; DC / AC power converters; Current mode control; Phase-Locked Loop.

\section{INTRODUÇÃO}

Atualmente, as usinas termelétricas são a segunda maior fonte produtora de energia, no Brasil, perdendo somente para as usinas hidrelétricas [1], o qual produzem energia em $\mathrm{CA}$, entretanto, estes tipos de fontes são poluentes, trazendo um impacto negativo ao meio ambiente [2], além disso podem não ser renováveis, quando se compara aos padrões de vida humano. Todavia, existem fontes não poluidoras em sua geração e também consideradas renováveis, como os painéis solares que produzem energia em CC [3], sendo necessário convertê-la em CA para permitir uma utilização mais abrangente, possibilitando a conexão destes sistemas ao sistema elétrico convencional.

Os equipamentos responsáveis por transformar a saída constante do painel solar em um sinal alternado são denominados de inversores de tensão tendo por finalidade interligar sistemas e dispositivos que funcionem em CA com fontes de geração em CC, estes funcionam a partir de um conjunto de dispositivos semicondutores de alta potência, como IGBT, que tem como função comutar repetidas vezes, de modo que o sinal constante na entrada do dispositivo possua variação periódica em sua saída, possibilitando obter uma função senoidal na saída [4][5].

Deste modo, o inversor tem um papel muito importante nos sistemas que envolvem a energia solar, pois o uso interligado a rede elétrica pode ocasionar alguns problemas para a concessionária, como: perda de coordenação em sistemas de proteção devido à sobrecorrentes, atuação indevida de religamentos instantâneos e operações em

\section{4 e 15 de março de 2019 Curitiba - Paraná}


ilhamento [6][7]. Com isto torna-se interessante utilizar métodos de controle para melhorar o desempenho deste dispositivo aplicado a painéis solares. Os tipos de controladores, são: controle de modo corrente e Phase-Locked Loop (PLL) [8][9][10].

\section{MODELAGEM DO INVERSOR DE TENSÃO}

O conversor CC-CA de tensão possui o sinal de saída alternado com valor médio nulo que pode ser gerado a partir de uma fonte independente constante como painéis solares [5]. A conversão do sinal CC é feita a partir de dois sinais modulantes, $m_{d}$ e $m_{q}$, que são grandezas constantes que compõem os três índices de modulação senoidais $m_{a}, m_{b}$ e $m_{c}$, esta transformação feita a partir da transformada inversa de Park. O modelo matemático apresentado neste artigo é baseado no modelo médio, visto que é possível descrever a relação entre a dinâmica da saída do inversor e os sinais de modulação utilizados, conforme apresentado na Eq. 1 [11].

$$
v_{t a}=\frac{V_{d c}}{2} m_{a} ; \quad v_{t b}=\frac{V_{d c}}{2} m_{b} ; \quad v_{t c}=\frac{V_{d c}}{2} m_{c}
$$

O conversor é conectado através de um filtro RL à rede elétrica padrão de energia, conforme mostrado na Fig. 1.

O conversor é conectado através de um filtro RL à rede elétrica padrão de energia, conforme mostrado no diagrama esquemático da Fig. 1.

Painel

Fotovoltáico

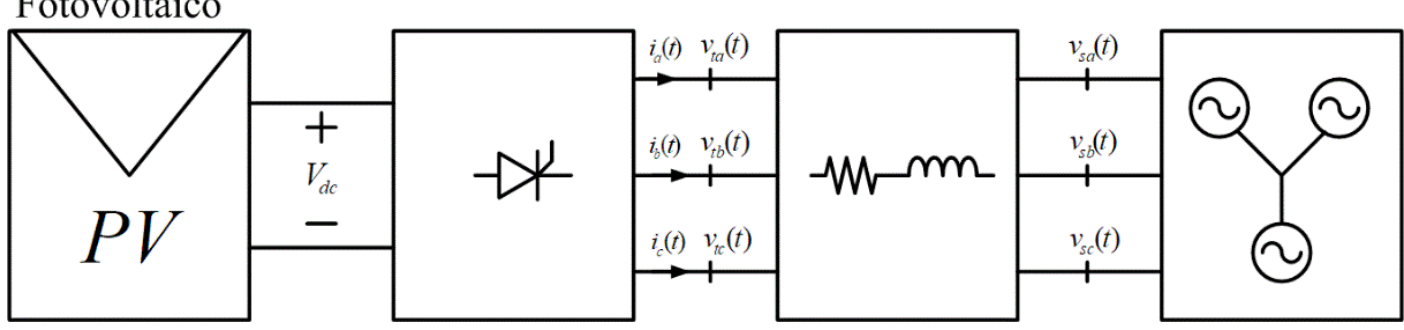

Figura 1: Diagrama da conexão do circuito analisado
Filtro $R L$

Rede Elétrica

q. 2

$$
\frac{i_{a}(t)}{v_{t a}(t)-v_{s a}(t)}=\frac{i_{b}(t)}{v_{t b}(t)-v_{s b}(t)}=\frac{i_{c}(t)}{v_{t c}(t)-v_{s c}(t)}=\frac{1}{L s+R}
$$

Para garantir o sincronismo de fase e frequência do sinal gerado pelo conversor com o sinal da rede elétrica, faz-se uso de um subsistema denominado de Phase-Locked Loop, que é implementado por meio de um controlador proporcional e integral, conforme apresentado na Eq. 3. Para a implementação do controle de potência transferida da entrada para a saída, faz-se uso de uma estrutura de controle PI, similar a apresentada pela Eq. 3, onde o sinal de controle é baseado na corrente elétrica do sistema.

$$
C(s)=\frac{k_{p} s+k_{i}}{s}
$$




\section{METODOLOGIA}

Para a implementação computacional das simulações dos controladores aplicados ao conversor CC-CA foi utilizado o software MATLAB/SIMULINK, de forma que foi possível realizar testes e avaliar o desempenho e funcionamento do sistema.

\section{Phase-Locked Loop (PLL)}

O PLL é um dispositivo que rastreia um sinal a partir de outro. Ele mantém o sinal de saída sincronizado com um sinal de referência [8]. O diagrama em blocos do controlador que é apresentado na Fig. 2. Seu objetivo é manter mínima a diferente entre $\rho=\omega_{0} t+\theta_{0}$.

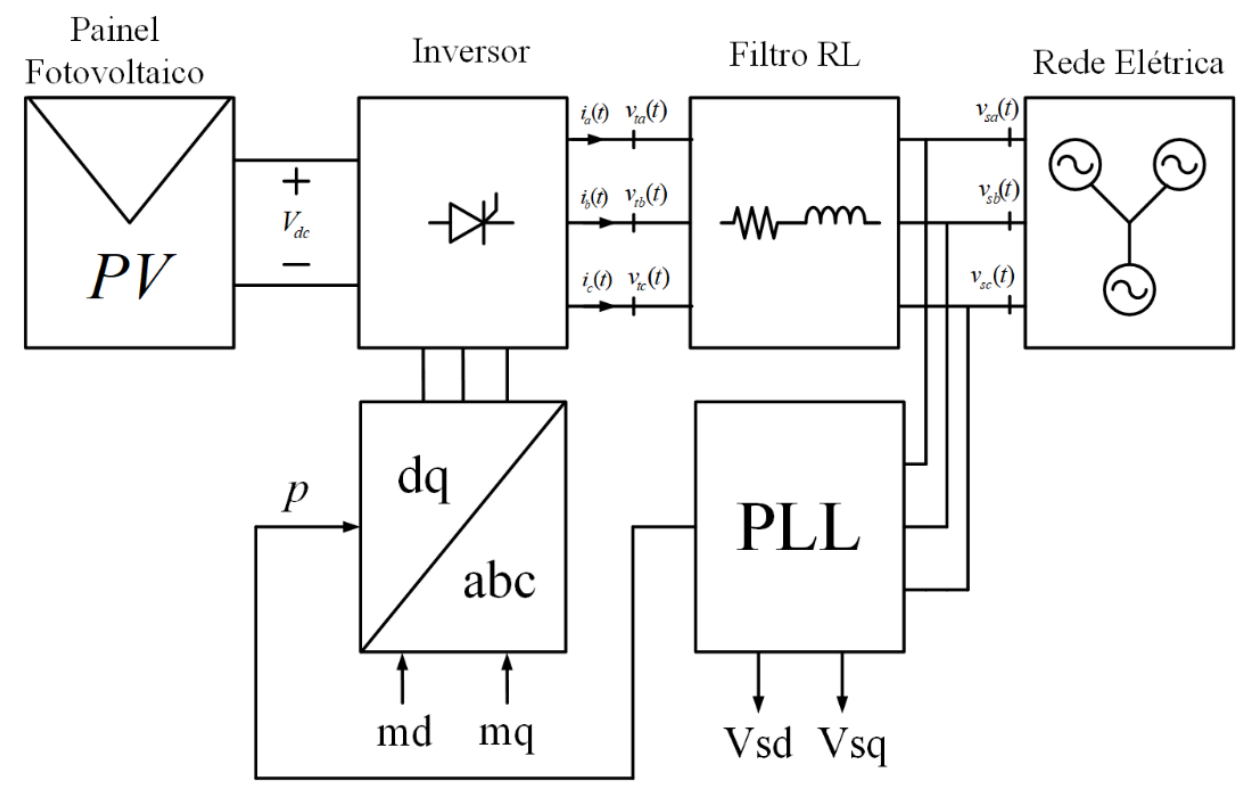

Figura 2: Diagrama em blocos do PLL

Utilizando o método do Lugar Geométrico das Raizes, LGR, [10], considerando a estrutura de controle do tipo PI, pode-se obter a Tabela 1, contendo os ganhos do controlador, cuja a estrutura foi apresentada na Eq. 3.

\section{Controle de corrente}

O controle das potências ativas e reativas fornecidas para a rede elétrica é feita por um controlador em modo corrente, sua escolha foi feita para proteger os sistemas contra sobrecorrentes, e também por ter como vantagem um desempenho dinâmico melhor e maior precisão de controle [11]. O diagrama em blocos do sistema de controle é apresentado na Fig. 3. Onde, $K_{d}(s)$ e $K_{q}(s)$ são duas estruturas de controle PI, projetados com o objetivo de aumentar o desempenho dinâmico do sistema e tornar o erro nulo de regime permanente. 


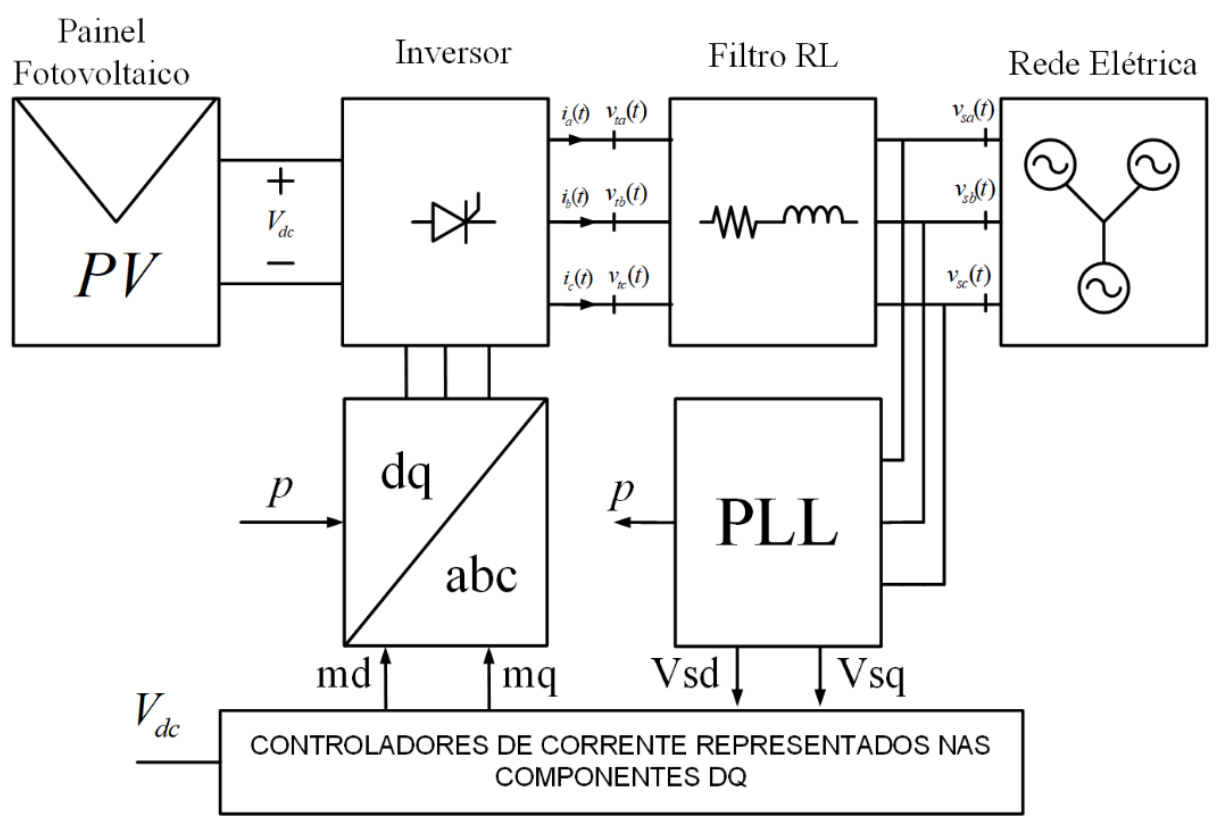

Figura 3: Diagrama em blocos do controle em modo corrente

Utilizando o método de cancelamento de polos e zeros, tem-se os seguintes valores dos ganhos na Eq. 8, para $\mathrm{R}=1,63 \mathrm{~m} \Omega$ e $\mathrm{L}=100 \mu \mathrm{H}$ e $\tau_{i}=1 \mathrm{~ms}$. o resultado dos valores dos ganhos do controlador na Tabela 1 . Sendo R e L a resistência e indutância da linha, respectivamente e $\tau_{i}$ é a constante de tempo em malha fechada.

$$
K_{p}=\frac{L}{\tau_{i}} ; \quad K_{i}=\frac{R}{\tau_{i}}
$$

Tabela 1: Resumo dos ganhos obtidos para o controlador PLL e o controlador em modo corrente

\begin{tabular}{c|c|c}
\hline Parâmetros & PLL & Modo corrente \\
\hline Método & LGR & Cancelamento de polos \\
\hline $\mathrm{K}_{\mathrm{p}}$ & 203 & 0,1 \\
$\mathrm{~K}_{\mathrm{i}}$ & 19361 & 1.63 \\
\hline
\end{tabular}

\section{Descrição dos testes realizados}

Para o controlador PLL, foram observadas as saídas das tensões nos eixos direto e de quadratura do modelo, e inserido um afundamento de tensão em uma das fases de $V_{s}$, com isto pode-se verificar o efeito deste afundamento no desempenho do PLL.

Para a realização do segundo experimento, realizou-se uma variação de degrau em $0.5 \mathrm{~s}$ e $0,7 \mathrm{~s}$ na referência de Id com variação de 0.7 p.u. para 0.5 p.u., em seguida no tempo de $0,9 \mathrm{~s}$ realizou-se uma nova variação de 0,25 p.u. na referência de Iq, considerando-se uma constante de tempo de acomodação de 5ms. Foi também inserida uma perturbação, objetivando avaliar o desempenho do sistema com os controles em modo de corrente. 


\section{RESULTADOS}

O modelo desenvolvido para o sistema, juntamente com o subsistema PLL e seus respectivos controladores, é apresentado na Fig. 4.

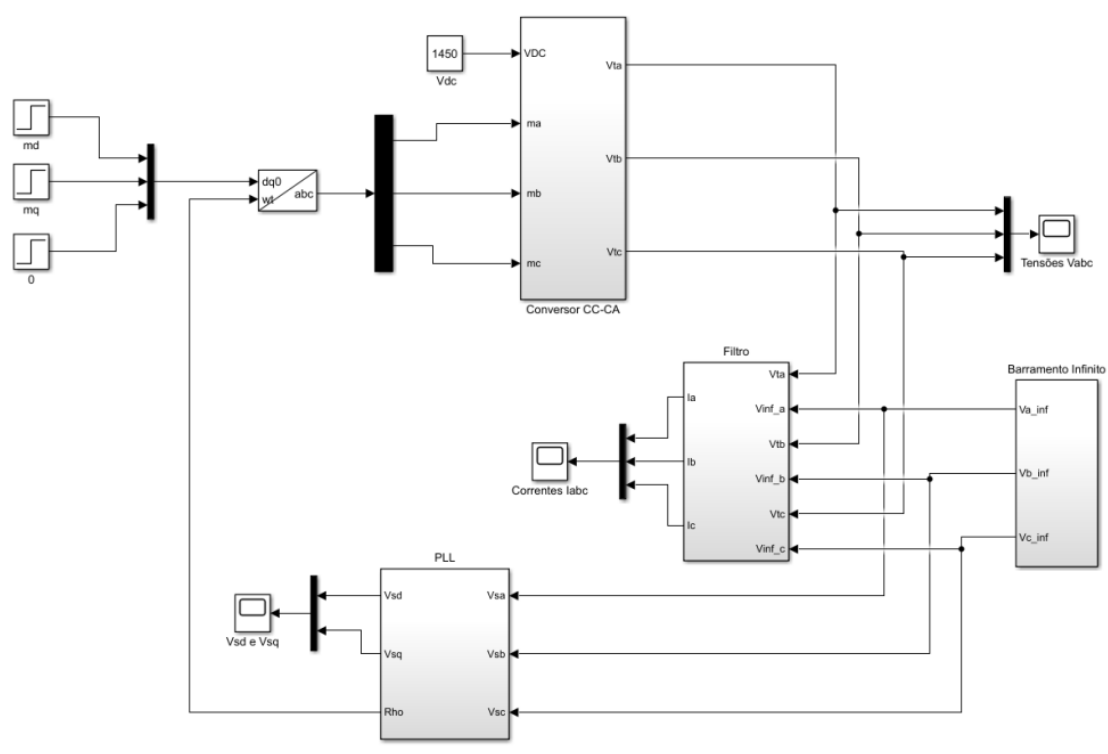

Figura 4: Modelo de simulação do sistema conectado à rede com PLL

A Fig. 5(a) apresenta a saída da tensão $V_{s}$ nos eixos direto e de quadratura do controlador PLL, atendendo as especificações de sobressinal não superior a $5 \%$ e um tempo de acomodação de $100 \mathrm{~ms}$.

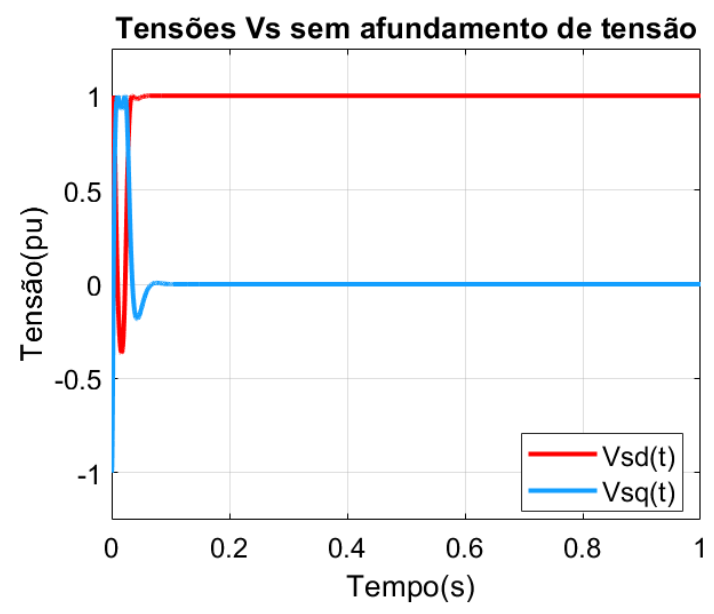

(a)

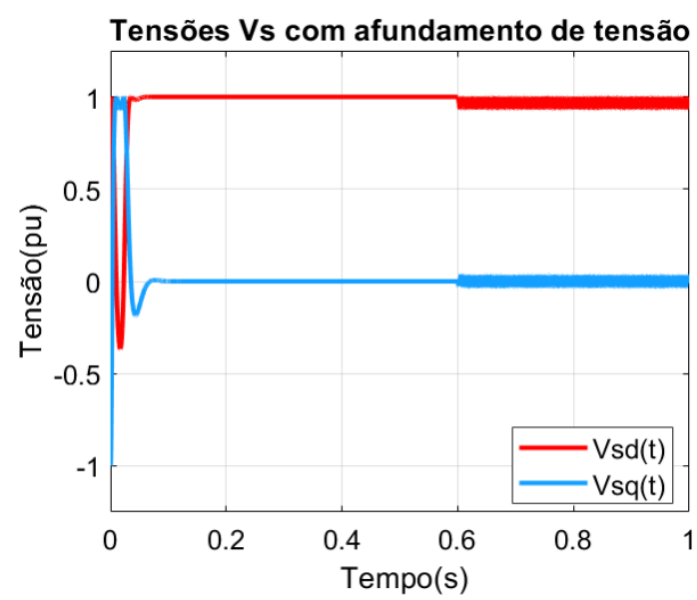

(b)

Figura 5: Tensões $V_{s}$ em pu da saída do controlador PLL

Vale ressaltar que quando se inclui um afundamento de tensão na fase $V_{s}$, no tempo de 0,6s, Fig. 5(b) há uma oscilação neste instante de tempo nas tensões $V_{s d}$ e $V_{s q}$, isto indica que a perturbação inserida provoca uma oscilação nos sinais do sistema para tentar manter o mesmo sob o setpoint de tensão previamente especificado. 


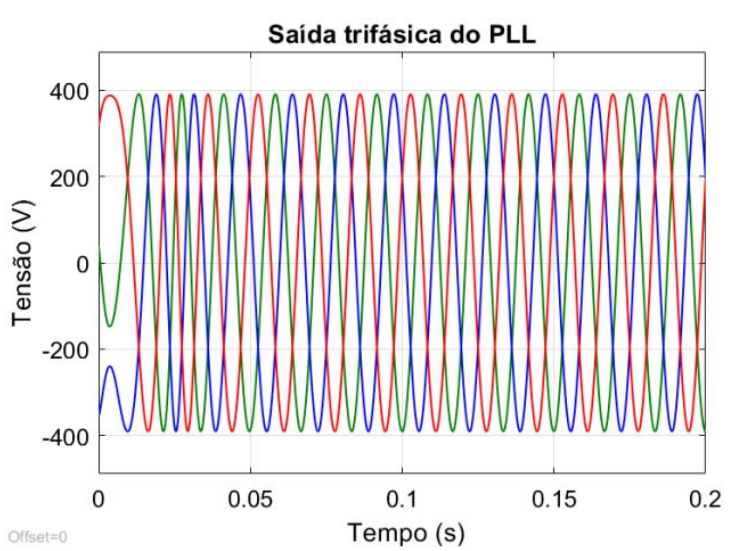

(a)

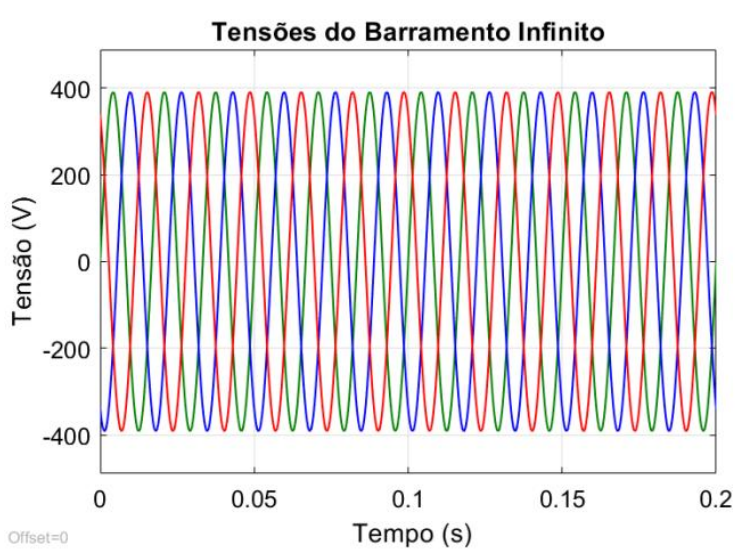

(b)

Figura 6: Comparação das tensões de saída com o barramento infinito

Na Fig. 6, tem-se um comparativo das saídas trifásicas do sistema. Pode-se observar que depois do período transitório a saída do PLL, Ffg 6(a), está equivalente as tensões trifásicas do barramento infinito, Fig 6(b).

Em seguida, foi incluída a implementação do controlador em modo corrente no ambiente computacional MATLAB/Simulink conforme é apresentado na Fig. 7, objetivando controlar o fluxo de potência a partir de um sinal de corrente.

A resposta do controlador em modo corrente apresentada na Fig. 8, apresenta o comportamento das correntes $I_{q}$ e $I_{d}$ de referência e a dinâmica das correntes, quando há mudança em suas respectivas referências. Para avaliar o desempenho do controlador, foi inserido um degrau de variação de referência nos tempos $0,5 \mathrm{~s}$ e $0,7 \mathrm{~s}$ para a corrente $I_{d}$, onde os valores de corrente variavam de 0,7 p.u. para 0,5 p.u. e de 0,5 p.u. para 0,9 p.u., respectivamente, em seguida realizou-se a variação de $I_{q}$ com variação de 0 a 0.25 p.u. em 0,9s. Pode-se observar que para cada variação de corrente, Fig. 8, o fluxo de potência transferido do conversor para a rede elétrica varia também, conforme é apresentado na Fig. 9.

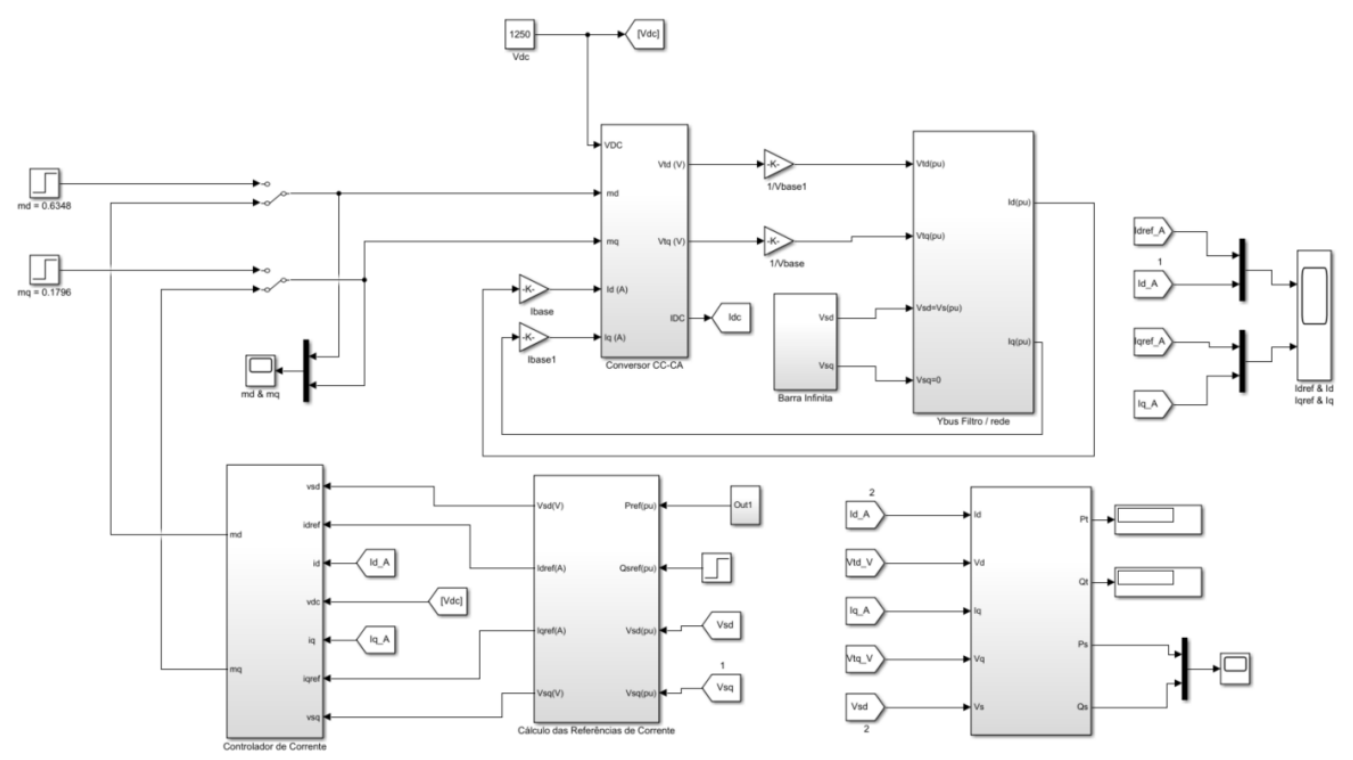

Figura 7: Implementação do controlador de corrente no ambiente computacional MATLAB/Simulink

Na Fig. 8 pode-se observar a compensação de todas as variações de referência das correntes de eixo direto e de quadratura, vale ressaltar que o controlador atendeu aos requisitos de desempenho demandados. 


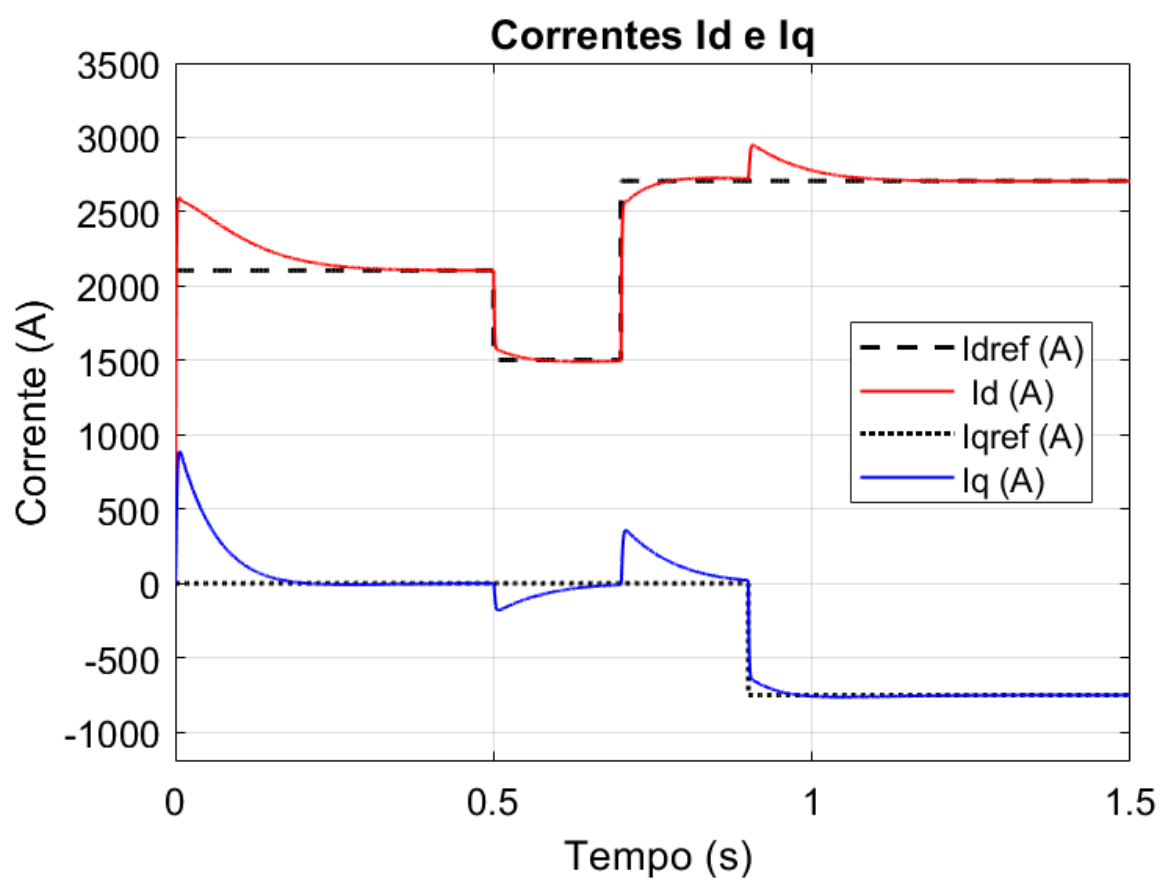

Figura 8: Correntes de eixo direto e de quadratura

No entanto, o ajuste do fluxo de potência ocorre conforme varia-se as referências de corrente, entretanto o controlador de potência ainda não apresentou o desempenho almejado, necessitando de ajustes para sua operação adequada, visto que o erro de saída nas potências está muito elevado.

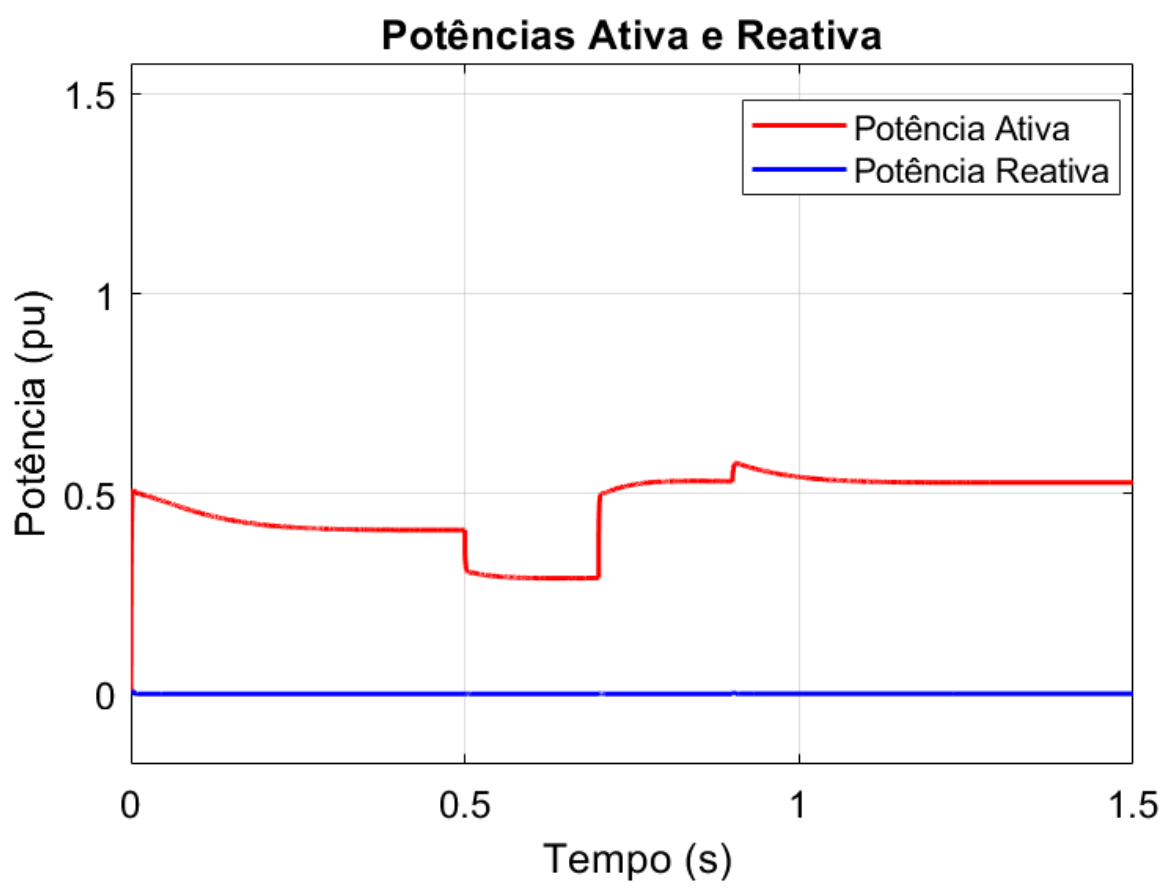

Figura 9: Potência Ativa e Reativa

\section{CONCLUSÃO}

Foi proposta a utilização de controlares para a melhoria do desempenho de conversores de tensão conectados a um barramento infinito. Os controlares avaliados foram o PLL e controle em modo corrente, ambos estruturados em controladores do tipo proporcional e integral. 
Os ganhos foram obtidos pelos métodos dos lugares das raízes e cancelamento de polos e zeros. Com isto, observou-se que com a inclusão dos controladores o desempenho do conversor pode ser melhorado de acordo com uma especificação desejada, o PLL cumpre o esperado ao manter o sincronismo do sinal de saída com o sinal de referência, ou seja a rede local de energia, e o controlador em modo corrente ajusta o fluxo de potência fornecida para a saída a partir de uma variação de corrente.

\section{REFERÊNCIA}

[1] BARROSO, L. A. Anuário Estatístico de Energia Elétrica 2017 ano base 2016. In: Empresa de Pesquisa Energética. Ministério de Minas e Energia. Rio de Janeiro, 2017

[2] TOLMASQUIM, M. T. Energia Renovável Hidráulica, Biomassa, Eólica, Solar, Oceânica In: EPE: Rio de Janeiro, 2016.

[3] VIllalva, M. G.; GAZOLI, J. R. Conceitos básicos. In: Energia Solar Fotovoltaica - Conceitos e Aplicações - Sistemas Isolados e Conectados à Rede. 1. ed. São Paulo: ERICA, 2012.

[4] HART, D. W., Power Eletronics. 1. ed. New York: McGraw-Hill, 2011. 494 p.

[5] BARBI, I., MARTINS, D. C., Introdução ao estudo dos conversores CC-CA, 2. Ed. Florianópolis: Ed dos Autores, 2008. 490p

[6] SOUZA, W. G., ROCHA, M. A, SERNI, P. J. A., ALVES, A. F., ANDREOLI, A. L., SILVA, P. S., Estudo de inversor aplicado a um Sistema Fotovoltaico para Compensação de Reativos. In: The 12th Latin-American Congress on Eletricity Generation and Transmission - CLAGTEE 2017

[7] JUNIOR, H. G., Estudos De Controladores Aplicados À Inversores Para UPS Com Operação Ilhada. Mestrado em engenharia elétrica, Universidade Estadual Paulista. 2013

[8] HSIEH, G. C., HUNG, J. C., Phase-Locked Loop Techniques - A Survey, IEEE Transaction On Industrial Eletronics, Vol 43, No. 6, December 1996.

[9] FERREIRA, R. J., ARAÚJO, R. E., PEÇAS LOPES, J. A., A comparative analysis and implementaion of various PLL techniques applied to single-phase grids. $3^{\text {rd }}$ International Youth Conference on Eergetics 2011, July.

[10] D'AZZO, HOUPIS, Análise e Projeto de Sistemas de Controle Lineares, ed. Guanabara Dois, 1978

[11] YAZDANI, A.; IRAVANI, R., Voltage-Sourced Converters in Power Systems: modeling, control, and applications, New Jersey: John Wiley \& Sons, 2010. 